\title{
Um índice de acessibilidade dos aeroportos que incorpora usuários com diferentes restrições de mobilidade
}

\author{
Lígia Gesteira Coelho ${ }^{1}$ e Antônio Nélson Rodrigues da Silva ${ }^{2}$
}

\begin{abstract}
Resumo: O objetivo deste trabalho é a criação de um índice de acessibilidade de aeroportos que considera vários grupos de usuários, incluindo estrangeiros e indivíduos com diferentes restrições de mobilidade. O método tem início com uma seleção de indicadores. Estes são agrupados de acordo com o modo de transporte disponível para acesso ao aeroporto: táxi, carona, automóvel próprio, automóvel de aluguel, ônibus regional e ônibus especial. Os indicadores são avaliados através de pesquisas e levantamentos de campo e recebem notas de acordo com o seu desempenho. Por fim, os escores dos indicadores são ponderados e uma nota final pode ser calculada para cada aeroporto. O maior valor encontrado em uma aplicação do índice a seis importantes aeroportos brasileiros foi 0,625 (em uma escala que vai de zero a um), no aeroporto Santos Dumont. Isto evidencia que há muitos aspectos a serem melhorados no que diz respeito à acessibilidade dos aeroportos no Brasil. DOI:10.4237/transportes.v20i3.596.

Palavras-chave: acessibilidade de aeroportos, índice de acessibilidade, restrições de mobilidade, indicadores de acessibilidade.
\end{abstract}

\begin{abstract}
The objective of this study is to create an index of accessibility to airports that takes into account several groups of users, including foreigners and persons with different mobility constraints. The method starts with a selection of indicators. They are grouped according to the transportation modes available for access to the airport: taxi, ride with a third party, own car, rental car, regional bus and shuttle bus. The indicators are evaluated through surveys and field observations and scored according to their performance. Finally, the scores are weighted and an overall score can be calculated for each airport. The highest value found in an application of the index to six major Brazilian airports was 0.625 (in a scale that goes from zero to one) for Santos Dumont Airport. The results highlight the fact that many aspects must still be improved regarding accessibility to Brazilian airports.

Keywords: airport accessibility, affordability index, restricted mobility, accessibility indicators.
\end{abstract}

\section{INTRODUÇÃO}

Os terminais aeroportuários vêm ampliando a sua importância como elementos estratégicos na definição da logística das pessoas e das mercadorias, tanto no contexto regional quanto no local. No contexto regional, eles passam a exercer um papel de escoamento de pessoas e mercadorias, tornando-se mais atrativos quanto maior for a distância da viagem. Já no contexto local, onde a sua operação naturalmente exerce maior impacto, sobressaem as características que levam a potencializar os problemas de mobilidade tão presentes na vida urbana.

No Brasil, segundo McKinsey \& Company (2010), viajam de avião mais de 110 milhões de passageiros ao ano, número que cresceu à taxa de $10 \%$ ao ano entre $2003 \mathrm{e}$ 2008, acompanhando a melhoria da economia como um todo e a inclusão de passageiros das classes B e C. Ao lado deste avanço da demanda, o crescimento recente trouxe uma nova série de desafios. A infraestrutura aeroportuária, em grande parte a cargo da Infraero, empresa que até 2012 administrava os aeroportos responsáveis por mais de $95 \%$ do tráfego aéreo civil, não cresceu no mesmo ritmo da demanda. Dos vinte principais aeroportos nacionais, treze já apresentam gargalos nos terminais de passageiros, com consequente redução no nível de serviço prestado aos usuários. Combinando-se o crescimento esperado da demanda

\footnotetext{
${ }^{1}$ Lígia Gesteira Coelho, Departamento de Engenharia de Transportes, Escola de Engenharia de São Carlos, Universidade de São Paulo, São Carlos, SP, Brasil. (e-mail: ligiagesteira@usp.br).

${ }^{2}$ Antônio Nélson Rodrigues da Silva, Departamento de Engenharia de Transportes, Escola de Engenharia de São Carlos, Universidade de São Paulo, São Carlos, SP, Brasil. (e-mail: anelson@sc.usp.br).
}

Manuscrito recebido em 13/7/2012 e aprovado para publicação em 27/11/2012. Este artigo é parte de TRANSPORTES v. 20, n. 3, 2012. ISSN: 2237-1346 (online). DOI:10.4237/transportes.v20i3.596. para os próximos 10 anos, conforme McKinsey \& Company (2010), em média de $5 \%$ ao ano, no cenário base, ou de até $7 \%$ ao ano, no mais otimista, com o fato de o parque aeroportuário já mostrar limitações, existe ainda o compromisso assumido pelo país de sediar dois eventos esportivos internacionais de grande porte: a Copa do Mundo de 2014 e as Olimpíadas de 2016.

São várias as ocasiões em que uma determinada característica operacional é tida como bem resolvida pelo administrador aeroportuário, enquanto pelo ponto de vista do usuário o serviço oferecido não é satisfatório. Em outras ocasiões, vistas pelo usuário, apresentam problemas sérios, mas que escapam da competência institucional do administrador, deixando a ele apenas a possibilidade de oficiar o órgão competente. E há questões nos dois lados desta equação, com parcela diretamente ligada ao administrador e outra fora de seu campo de ação, como é o caso da mobilidade dos passageiros. Parte de suas viagens em terra ocorre em território sob o controle da Infraero e o restante, que é diretamente proporcional à localização do aeroporto no contexto urbano, sob a gestão de autoridades sem ligação com o setor aéreo.

Além destes aspectos, há ainda a necessidade de quantificar os instrumentos de avaliação, de forma a diminuir eventuais problemas de falta de clareza e consistência dos mesmos. Assim, o objetivo deste trabalho é a criação e aplicação de um índice de acessibilidade de aeroportos com base em aspectos objetivos, quantificáveis, que permita fazer comparações diretas entre os terminais estudados. A partir de uma perspectiva de avaliação competitiva entre as soluções encontradas pelos diversos aeroportos para os desafios de sua acessibilidade, pretende-se apontar as melhores práticas, estabelecendo um sistema de benchmarking na busca de um desempenho superior. Pretende-se estabelecer 
um processo positivo e pró-ativo que permita que o operador de um terminal examine como outro realiza uma função específica a fím de incorporá-la ao seu contexto e melhorar a sua própria condição.

\section{ACESSIBILIDADE DOS AEROPORTOS}

Embora seja possível definir acessibilidade de forma simples e direta, como sendo a facilidade de se chegar ao destino desejado, o conceito pode dar origem a várias abordagens quando se tenta dimensioná-lo. A distância, por exemplo, pode a princípio ser uma boa medida de acessibilidade, porém em sistemas congestionados o tempo de viagem pode ser mais apropriado. Por outro lado, deve-se verificar para quem está direcionada esta "facilidade de se chegar ao destino desejado". Além disso, quando se pensa em uma pessoa com dificuldades específicas de locomoção, a questão pode ter um outro entendimento. A NBR 14273, intitulada ACESSIBILIDADE DA PESSOA PORTADORA DE DEFICIÊNCIA NO TRANSPORTE AÉREO COMERCIAL, por exemplo, especifica mais restritamente o conceito ao definir acessibilidade como "possibilidade e condição de alcance pela pessoa portadora de deficiência para utilização com segurança e, quando aplicável, com autonomia, de espaços aeroportuários e aeronaves" (ABNT, 1999).

De modo geral pode-se entender que o objetivo previsto quando se melhora a acessibilidade é o de permitir e facilitar o acesso aos serviços, produtos e atividades que são de interesse de determinado grupo de pessoas. Quando uma análise se concentra no transporte aéreo, especificamente nas questões de acessibilidade da viagem até o aeroporto ou entre este e o destino final na cidade (trabalho, residência, etc.), foco deste estudo, verifica-se uma série de variáveis adicionais que tem influência na avaliação objetiva de acessibilidade que o usuário utiliza para fazer as suas escolhas de viagem. De acordo com Caves e Gosling (1999), uma vez que o indivíduo identifica a necessidade de realizar uma viagem, imediatamente faz a escolha do modo de transporte ou combinação de modos a ser feita. Se for pelo transporte aéreo, haverá um conjunto adicional de decisões a serem realizadas, tais como a do aeroporto, a do serviço (táxi aéreo ou aviação regular), a da companhia, da rota, entre outras.

Quando se trata da escolha do aeroporto, nos casos em que há mais de um competindo em uma região, inúmeros autores apontam a facilidade de acesso como um dos fatores decisórios básicos, com destaque para Skinner (1976) e Harvey (1986). Aumentar a acessibilidade de um terminal pode ser, portanto, em um cenário de disputa entre terminais, uma estratégia para incrementar o número de usuários. São Francisco, cidade nos Estados Unidos servida por três importantes aeroportos, é frequentemente selecionada para a realização deste tipo de estudo. Monteiro e Hansen (1996), por exemplo, verificaram os efeitos da introdução de melhorias na acessibilidade do lado terrestre sobre a participação no mercado destes aeroportos. Pels et al. (2001), ainda no mesmo cenário regional, concluíram que tanto viajantes a negócios como a lazer escolhem primeiro o aeroporto e depois a companhia aérea. Isso sugere que talvez o viajante esteja mais propenso a alternar a companhia do que o aeroporto. Pels et al. (2001) demonstram ainda que investimentos na acessibilidade de um aeroporto resultam em benefícios tanto para o aeroporto quanto para as companhi- as aéreas.

Para Kouwenhoven (2008), mudanças na acessibilidade de um ou mais aeroportos podem ter grande efeito na sua escolha pelos passageiros, especialmente se não há diferenças significativas entre as instalações internas oferecidas nos terminais concorrentes. Dentre os fatores que podem influenciar na escolha do aeroporto, estão: disponibilidade de voos para o destino específico e da companhia aérea preferida, frequência dos voos, preço da passagem, tempo de voo, qualidade dos voos (serviço de bordo, pontualidade), facilidade para fazer o check in, serviços oferecidos (shopping, restaurantes), instalações para bagagens e imigração e acessibilidade do aeroporto. Para a escolha do modo de acesso, os fatores são: disponibilidade dos modos de acesso, tempo de acesso, custo, frequência (no caso de transporte público), conforto e confiabilidade. Em estudo nos Países Baixos (2008), o mesmo autor adota o custo generalizado do acesso ao aeroporto como indicador de acessibilidade.

Para Gosling (1997), embora muitos defendam que aprimorar o acesso ao aeroporto envolve dotá-lo de ligações ferroviárias de alta capacidade, os altos investimentos em geral associados aos modos ferroviários raramente são justificados pela demanda existente. Em outro estudo, Gosling (2008) faz uma revisão crítica dos modelos de acesso a aeroportos e cita estratégias alternativas para melhorar a atratividade para os passageiros, como o uso de terminais remotos do lado terrestre, localizados em pontos da cidade com grande concentração de usuários. Neste caso, as bagagens podem ser despachadas e o acesso ao aeroporto realizado por ônibus expresso, solução que tem se mostrado efetiva em algumas grandes metrópoles.

\subsection{Fatores que influenciam a acessibilidade}

De modo geral verifica-se que a busca para a solução da acessibilidade de aeroportos é complexa e não é resolvida através de um único atributo. Para Harvey (1986), embora tempo e custo da viagem sejam muito importantes na escolha do modo de acesso, outros fatores, como o número de bagagens carregadas, por exemplo, são também relevantes. Clark e Lam (1990) destacam também a importância do motivo da viagem e da região de residência do passageiro. Em uma pesquisa com maioria de jovens do sexo masculino, Mamede e Alves (2009) demonstraram que os usuários decidem o modo de viagem considerando um conjunto de fatores. identificaram "rapidez" como o atributo mais importante. Em seguida, vieram "segurança" e "custo", classificados quase com o mesmo valor e, por último, o "conforto".

O tipo de usuário, diferenciado, por exemplo, pela sua experiência no uso do modo aéreo, é um fator importante a ser considerado quando da análise da escolha do modo para o acesso ao aeroporto. Koster et al. (2010) mostram que aqueles que viajam entre 5 e 10 vezes por ano optam por chegar, em média, 10 minutos depois do que aqueles com menos experiência. Já viajantes que voam mais de 10 vezes por ano chegam até 20 minutos mais tarde aos terminais. Em outra pesquisa, conduzida em 2001 no aeroporto de Heathrow, em Londres, Kouwenhoven (2008) demonstrou ainda que não residentes têm pequena participação de uso de automóveis e tendem a usar o transporte público mais intensamente. Os que viajam por motivo negócios, em geral, têm uma forte preferência por utilizar serviços de táxi, uma vez que estão dispostos a pagar mais com o intuito de 
economizar no tempo de viagem. Para usuários com dificuldade de locomoção, como cadeirantes e deficientes visuais, um aspecto importante é se o aeroporto possui serviços que sejam adaptados às suas necessidades.

\subsection{Acessibilidade de aeroportos brasileiros}

No Brasil este tipo de competição entre aeroportos na mesma região que poderia acelerar as soluções de acessibilidade acontece raramente. Mesmo na ocorrência de mais de um aeroporto por região, normalmente eles exercem funções complementares, que não configuram um ambiente de disputa pelo cliente. Outra dificuldade na análise é o fato de aeroportos situados em diferentes pontos de uma região oferecerem, e carecerem, de diferentes condições de acesso. Aqueles nas proximidades dos centros urbanos têm necessidades diversas daqueles com uma localização mais periférica, como pode ser facilmente verificado quando se analisa os conjuntos Congonhas - Guarulhos (e de forma mais intensa no passado recente, Viracopos), Santos Dumont Galeão e Pampulha - Confins. Nos casos de Congonhas, Santos Dumont e Pampulha, as dificuldades se devem sobretudo às barreiras criadas pelos congestionamentos que o tráfego da cidade impõe ao entorno do aeroporto. Os outros, mais retirados, sofrem pela falta de soluções viárias e de transporte público que aumentem as opções, ao mesmo tempo em que reduzam e regularizem o tempo de viagem.

Deste modo, se por um lado a localização do aeroporto perto das áreas centrais da cidade favorece um conjunto de diferentes polos de viagem reduzindo os deslocamentos, esta posição relativa gera conflitos ao compartilhar os principais corredores de tráfego da cidade com as suas viagens rotineiras de diversos destinos e alta intensidade. Dentro deste contexto, os estudos de acessibilidade de aeroportos no Brasil são normalmente direcionados a explicar os fatores que influenciam a escolha do modo até o aeroporto e no que poderia melhorar a acessibilidade de um aeroporto. Alves (2005), em um estudo detalhado para o Aeroporto Internacional André Franco Montoro, em Guarulhos, através de uma pesquisa de preferência declarada apenas com residentes da região metropolitana de São Paulo, mostra a importância do fator variabilidade do tempo de viagem na escolha modal de acesso terrestre. Braga e Guedes (2008) criaram um método, utilizando um Sistema de Informações Geográficas (SIG), para ordenar os oito principais aeroportos brasileiros em relação à facilidade de se chegar a eles por via terrestre. Após esta classificação, apontaram medidas que poderiam melhorar as condições de acessibilidade do Aeroporto Internacional Tancredo Neves. No entanto, neste estudo o único aspecto considerado foi o tempo de viagem entre o centro da cidade e o aeroporto, para os modos rodoviário e ferroviário.

\section{MÉTODO}

O método para criação do Índice de Acessibilidade a ser aplicado nos aeroportos brasileiros foi desenvolvido em três etapas. Realizou-se, inicialmente, um levantamento do "estado da arte", através do qual uma série de indicadores foi listada. Esses indicadores surgiram através da identificação de potenciais problemas que os passageiros podem enfrentar ao acessar o aeroporto e foram classificados em seis grandes grupos, de acordo com o modo de transporte disponível para o usuário no acesso até o aeroporto: táxi, ca- rona, automóvel próprio, aluguel de automóvel, ônibus regional e ônibus especial. Então, os indicadores foram avaliados através de pesquisas e levantamentos de campo nos aeroportos estudados e receberam notas de acordo com o seu desempenho. Por fim, esses indicadores foram ponderados e a nota final de cada aeroporto pôde ser calculada.

Para aplicação e avaliação do índice proposto foram escolhidos os aeroportos das cidades brasileiras onde o movimento de passageiros é muito expressivo: São Paulo, Rio de Janeiro, Belo Horizonte e Brasília. Juntos, esses aeroportos representam quase $75 \%$ do movimento de passageiros do país. No entanto, as pesquisas utilizadas neste estudo não incluíam os aeroportos de Belo Horizonte. Pampulha e Confins foram, portanto, excluídos desse estudo. Os aeroportos analisados foram: em São Paulo, Congonhas, Guarulhos e Viracopos, no Rio de Janeiro, Galeão e Santos Dumont, e em Brasília, Juscelino Kubitschek.

\subsection{Tipos de usuários}

Para fins de classificação, os passageiros foram divididos em duas categorias principais em função de suas condições de mobilidade: plena e reduzida. Dentro da primeira categoria está configurado apenas o passageiro sem bagagens, adulto, e que não apresenta nenhuma dificuldade de locomoção. Dentro da categoria reduzida, estão os estrangeiros - com dificuldades específicas de locomoção decorrentes da dificuldade de comunicação em função da linguagem, o deficiente visual, o cadeirante, a gestante, o idoso. Insere-se ainda nessa categoria o passageiro com bagagens, que sofre restrições inerentes ao volume adicional que carrega, dificultando o uso de alguns modos de transporte e com sérias restrições no que diz respeito a longos caminhamentos.

\subsection{Indicadores}

A abordagem sobre a qualidade da acessibilidade dos aeroportos vai então seguir uma estrutura que parte da análise do atendimento ao passageiro comum, adulto e sem bagagem (ou somente com bagagem de mão). Estas configuram as condições mínimas a serem oferecidas pelo aeroporto, já que representa o usuário que tem todas as capacidades - físicas e intelectuais - necessárias para realizar o deslocamento. O passo seguinte é verificar as alterações que se processam quando este usuário estiver com bagagens. As dificuldades específicas e as adequações necessárias serão, então, avaliadas. A partir daí, sempre considerando as dificuldades adicionais de cada tipo de usuário, viriam as categorias "gestantes e idosos", "estrangeiros", "cadeirantes" e "deficientes visuais".

Os indicadores são avaliados como uma estrutura incremental, a partir das exigências dos usuários sem bagagens. Estas seriam as características mínimas exigidas para um terminal e que, se não atendidas para este tipo de usuário, com certeza terão impactos ainda mais negativos para aqueles que não estão dotados de sua capacidade integral. Os indicadores considerados para cada tipo de usuário são mostrados na Tabela 1. As diferentes categorias de usuários podem exigir adaptações cada vez mais específicas, muitas vezes não demandadas pela categoria "passageiros sem bagagem", tal como se pode extrair da análise da referida tabela.

Para o desenvolvimento do estudo as disponibilidades de serviços oferecidos e a verificação de suas dimensões, as condições locais serão confrontadas com as práticas nacio- 
Tabela 1. Indicadores considerados para os diferentes tipos de usuários dos aeroportos

\begin{tabular}{|c|c|c|c|c|c|c|}
\hline \multirow[b]{2}{*}{ INDICADORES } & \multicolumn{6}{|c|}{ PASSAGEIROS } \\
\hline & $\begin{array}{l}\text { Sem } \\
\text { bagagem }\end{array}$ & $\begin{array}{l}\text { Com } \\
\text { bagagem }\end{array}$ & $\begin{array}{l}\text { Gestantes } \\
\text { e idosos }\end{array}$ & Estrangeiros & Cadeirantes & $\begin{array}{l}\text { Deficientes } \\
\text { visuais }\end{array}$ \\
\hline Tempo e custo & $\mathrm{X}$ & $\mathrm{X}$ & $\mathrm{X}$ & $\mathrm{X}$ & $\mathrm{X}$ & $\mathrm{X}$ \\
\hline Sinalização & $\mathrm{X}$ & $\mathrm{X}$ & $\mathrm{X}$ & $\mathrm{X}$ & $\mathrm{X}$ & $\mathrm{X}$ \\
\hline Meio fio & $\mathrm{X}$ & $\mathrm{X}$ & $\mathrm{X}$ & $\mathrm{X}$ & $\mathrm{X}$ & $\mathrm{X}$ \\
\hline $\begin{array}{l}\text { Distância das vagas do meio fio até o terminal } \\
\text { de passageiros }\end{array}$ & $\mathrm{X}$ & $\mathrm{X}$ & $\mathrm{X}$ & $\mathrm{X}$ & $\mathrm{X}$ & $\mathrm{X}$ \\
\hline Pré pagamento de táxi & $\mathrm{X}$ & $\mathrm{X}$ & $\mathrm{X}$ & $\mathrm{X}$ & $\mathrm{X}$ & $\mathrm{X}$ \\
\hline Número de vagas no estacionamento & $\mathrm{X}$ & $\mathrm{X}$ & $\mathrm{X}$ & $\mathrm{X}$ & $\mathrm{X}$ & $\mathrm{X}$ \\
\hline Preço do estacionamento & $\mathrm{X}$ & $\mathrm{X}$ & $\mathrm{X}$ & $\mathrm{X}$ & $\mathrm{X}$ & $\mathrm{X}$ \\
\hline Vaga e caminho coberto & $\mathrm{X}$ & $\mathrm{X}$ & $\mathrm{X}$ & $\mathrm{X}$ & $\mathrm{X}$ & $\mathrm{X}$ \\
\hline $\begin{array}{l}\text { Distâncias percorridas } \\
\text { até o terminal }\end{array}$ & $\mathrm{X}$ & $\mathrm{X}$ & $\mathrm{X}$ & $\mathrm{X}$ & $\mathrm{X}$ & $\mathrm{X}$ \\
\hline $\begin{array}{l}\text { Condições de conforto nos Pontos de } \\
\text { Embarque e Desembarque (PEDs) }\end{array}$ & $\mathrm{X}$ & $\mathrm{X}$ & $\mathrm{X}$ & $\mathrm{X}$ & $\mathrm{X}$ & $\mathrm{X}$ \\
\hline $\begin{array}{l}\text { Caminho coberto do ponto de ônibus até o } \\
\text { terminal }\end{array}$ & $\mathrm{X}$ & $\mathrm{X}$ & $\mathrm{X}$ & $\mathrm{X}$ & $\mathrm{X}$ & $\mathrm{X}$ \\
\hline Número de linhas que atendem o aeroporto & $\mathrm{X}$ & $\mathrm{X}$ & $\mathrm{X}$ & $\mathrm{X}$ & $\mathrm{X}$ & $\mathrm{X}$ \\
\hline Faixa de pedestres & & $\mathrm{X}$ & $\mathrm{X}$ & $\mathrm{X}$ & $\mathrm{X}$ & $\mathrm{X}$ \\
\hline Qualidade da calçada & & $\mathrm{X}$ & $\mathrm{X}$ & $\mathrm{X}$ & $\mathrm{X}$ & $\mathrm{X}$ \\
\hline $\begin{array}{l}\text { Número de vagas específicas no } \\
\text { estacionamento }\end{array}$ & & & $\mathrm{X}$ & & $\mathrm{X}$ & \\
\hline Sinalização & & & & $\mathrm{X}$ & & \\
\hline Atendimento em inglês & & & & $\mathrm{X}$ & & \\
\hline Meio fio reservado pra deficiente & & & & & $\mathrm{X}$ & \\
\hline $\begin{array}{l}\text { Distância do ponto até o terminal de } \\
\text { passageiros }\end{array}$ & & & & & $\mathrm{X}$ & \\
\hline Rebaixo nas vagas do meio fio & & & & & $\mathrm{X}$ & \\
\hline Entrada e saída adaptada & & & & & $\mathrm{X}$ & \\
\hline Veículo adaptado para cadeirante & & & & & $\mathrm{X}$ & \\
\hline Linha guia ou piso tátil & & & & & & $\mathrm{X}$ \\
\hline
\end{tabular}

nais e internacionais de modo a permitir avaliar a sua importância relativa no conjunto de alternativas de atendimento ao usuário. Esta aproximação permite, em primeiro lugar, analisar o terminal do ponto de vista da oferta, avaliando a sua estrutura no que diz respeito à quantidade dos serviços oferecidos. A verificação final da qualidade dos serviços oferecidos será exercida sob a perspectiva de cada uma das categorias de usuários, configurando deste modo o exercício final que é objetivo desse trabalho.

\subsubsection{Passageiro sem bagagem}

\section{A. Tempo e custo}

Através das pesquisas realizadas em outros estudos (por exemplo, FIPE, 2009), foi calculado o tempo e o custo médio de acesso da origem do usuário até o aeroporto em questão para todos os meios de transportes avaliados. Embora os valores levantados pela pesquisa sejam do período de julho a setembro de 2009, para o objetivo de estabelecer um ranking para a acessibilidade dos aeroportos brasileiros, eles são adequados. De maneira a tornar os dados mais atualizados possíveis, foram calculados novos valores (para fevereiro de 2012), corrigidos pelo IPCA (Índice de Preços ao Consumidor Amplo) acumulado do período, que é medido mensalmente pelo IBGE (Instituto Brasileiro de Geografia e Estatística).

\section{B. Sinalização}

A sinalização indicativa tem por finalidade indicar as vias e orientar os condutores de veículos até o aeroporto. Para esse indicador, foi verificado se a sinalização é suficiente e adequada para atingir o objetivo de permitir que um moto- rista que não conhece o aeroporto possa se localizar naquele ambiente. Ela deve ser clara, eficiente e apresentar um bom estado de conservação. Os aeroportos que atendem a esses quesitos foram avaliados, através de levantamento, com a nota máxima 1 (um).

\section{Meio-fio}

Para análise do comprimento do meio-fio deste trabalho foi escolhido o método da IATA (1989). Após o cálculo do comprimento necessário de meio-fio para uso dos táxis e automóveis, foi feita uma análise que consistiu em calcular a razão da disponibilidade existente sobre aquela necessária. Os cálculos foram feitos separadamente para o meio-fio de embarque e desembarque.

\section{Distância das vagas do meio-fio até o terminal de pas- sageiros}

Para o cálculo desse indicador, foi medida a distância do centro geométrico do conjunto de vagas até o acesso mais próximo ao terminal. Para aeroportos que operam com mais de um posicionamento para táxi e/ou embarque e desembarque de automóveis, foi feita a média ponderada de acordo com o número de vagas.

Dessa forma, foram consideradas as seguintes informações:

$V_{m}=$ Número total de vagas contínuas destinadas a cada modo de transporte;

$D_{p}=$ Distância do centro geométrico do conjunto de vagas contínuas até o acesso mais próximo ao terminal para cada modo de transporte; 


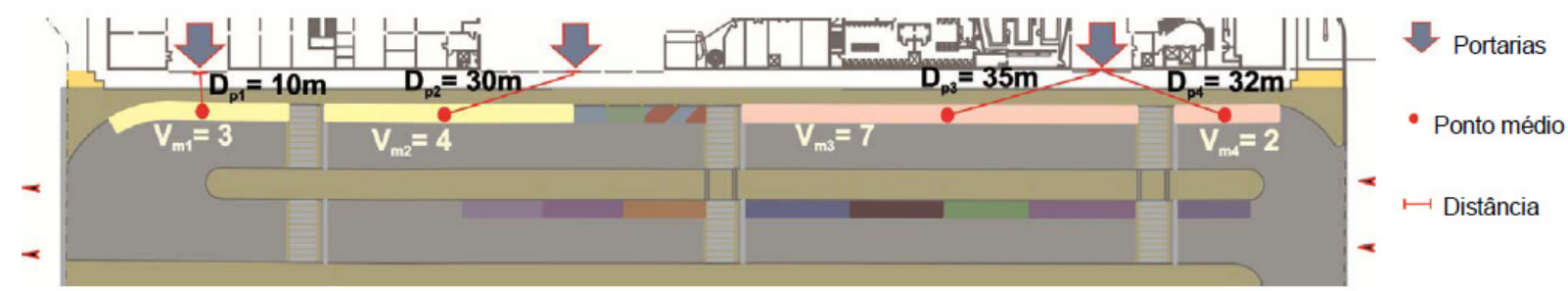

Figura 1. Exemplo de cálculo da distância das vagas do meio-fio até o terminal de passageiros aplicado ao setor de desembarque do Aeroporto Santos Dumont - Rio de Janeiro

$D_{T}=$ Distância média das vagas do meio-fio até o terminal de passageiros para cada modo de transporte.

Com esses dados, encontra-se a Distância média das vagas do meio-fio $\left(\mathrm{D}_{\mathrm{T}}\right)$, através do cálculo da média ponderada, conforme a equação (1).

$$
D_{T}=\frac{\sum\left(V_{m} \cdot D_{p}\right)}{\sum V_{m}}
$$

A seguir foi utilizado um exemplo (Figura 1) para explicação da forma de cálculo do parâmetro $\mathrm{D}_{\text {T. }}$ Embora baseado no terminal de desembarque do Aeroporto Santos Dumont, os dados de distância e quantidade de vagas são fictícios, usados somente para fins de exemplificação.

$$
\begin{aligned}
D_{T} & =\frac{\left(V_{m 1} \cdot D_{p 1}\right)+\left(V_{m 2} \cdot D_{p 2}\right)+\left(V_{m 3} \cdot D_{p 3}\right)+\left(V_{m 4} \cdot D_{p 4}\right)}{V_{m 1}+V_{m 2}+V_{m 3}+V_{m 4}} \\
D_{T} & =\frac{(3 \cdot 10)+(4 \cdot 30)+(7 \cdot 35)+(2 \cdot 32)}{3+4+7+2} \\
D_{T} & =\frac{459}{16} \\
D_{T} & =28,6 m
\end{aligned}
$$

\section{E. Pré-pagamento}

Nesse indicador é verificado se as empresas de táxi que atendem o aeroporto possuem a opção de pré-pagamento. Aeroportos que possuem essa opção foram pontuados com a nota 1 (um), enquanto os aeroportos que não possuem foram pontuados com 0 (zero).

\section{F. Número de vagas no estacionamento}

Para análise do número de vagas necessárias no estacionamento, optou-se por adotar o índice da FAA (1988) que considera 1,5 vagas por passageiro na hora pico e é um índice usado internacionalmente. Após o cálculo do número de vagas necessário, foi estabelecida a razão entre esse número e as vagas existentes.

\section{G. Preço do estacionamento}

$\mathrm{O}$ estacionamento do aeroporto serve tanto para acompanhantes que deixam seus automóveis por períodos curtos, de até 3 horas, quanto para passageiros que deixam seus veículos no aeroporto por longos períodos enquanto viajam. Quanto menor esse preço cobrado, melhor para o usuário. Esse indicador avalia o preço cobrado pela primeira hora de estacionamento.

\section{H. Vaga e caminho coberto}

Nesse indicador foi verificado se as vagas e o caminho do estacionamento até o terminal possuem cobertura. Estacionamentos que possuem todas as vagas cobertas foram pontuados com 1 (um), enquanto os que possuem todas as vagas descobertas pontuados com 0 (zero). No caso de apenas algumas vagas serem cobertas, foi feita uma proporção com o número total de vagas.

\section{Distâncias percorridas até o terminal}

A distância aqui considerada é aquela que o usuário tem que percorrer entre o seu automóvel próprio deixado no estacionamento ou aquela entre o ponto de ônibus regular até a entrada mais próxima do terminal de passageiros. Para aqueles que dispõem de mais de um ponto de ônibus, foi feita uma média dessas distâncias.

\section{J. Condiçães de conforto dos PEDs (Pontos de Embarque e Desembarque)}

Os pontos de embarque e desembarque de cada aeroporto foram avaliados separadamente, cada um obtendo como nota máxima 1 (zero). Essa nota originou-se da soma da avaliação das condições do PED, sendo distribuídas da seguinte forma: $34 \%$ da nota se o PED possuir abrigo, $33 \%$ se houver assento e os outros $33 \%$ se existir sinalização. Após a análise de todos os PEDs relativos ao aeroporto, foi feita uma média para obter a nota final da situação dos PEDs do aeroporto.

\section{K. Caminho coberto do ponto de ônibus até o terminal}

Para os passageiros que chegam ou partem do aeroporto utilizando o transporte coletivo, é importante que haja proteção contra variações do ambiente no percurso do ponto de embarque / desembarque até o terminal de passageiros. Aeroportos que possuem essa cobertura são avaliados com a nota máxima 1 (um) enquanto os que não possuem foram avaliados com 0 (zero).

\section{Número de linhas que atendem o aeroporto}

Supõe-se que as linhas de ônibus urbano que atendem ao aeroporto o interligam a diferentes regiões da cidade. Foi considerado, portanto, que quanto maior o número de linhas de ônibus que passam pelo aeroporto, mais facilmente serão realizados os deslocamentos para os diversos pontos da cidade. Esse indicador avalia se o aeroporto é bem atendido pelo transporte público urbano.

\subsubsection{Passageiro com bagagem}

\section{A. Faixa de pedestres}

Nesse indicador, é verificada a ocorrência das faixas de pedestres em frente aos terminais, se elas são bem sinalizadas, se existem rampas e meio-fio rebaixados, facilitando tanto o carregamento de bagagens como o deslocamento de usuários com restrições de mobilidade. Os aeroportos que atendem a esses requisitos foram avaliados com pontuação de 0 
(zero) a 1 (um), obedecendo a seguinte distribuição: $34 \%$ referentes ao parâmetro de existência da faixa de pedestres, $23 \%$ referentes às condições da sinalização horizontal da mesma, $10 \%$ referentes à existência de sinalização vertical e $33 \%$ considerada a existência de rebaixos e rampas. Os que não atendem receberam pontuação 0 .

\section{B. Qualidade da calçada}

Nesse indicador foi avaliado o estado de conservação da calçada entre o estacionamento e o terminal de passageiros, se existem buracos e desníveis nos acessos e se a circulação de passageiros com malas e de usuários com restrições de mobilidade pode ser feita com conforto.

As calçadas de cada aeroporto foram analisadas separadamente, cada um obtendo como nota máxima o valor 1 (um). Essa nota originou-se da soma da avaliação das condições da calçada, sendo distribuídas da seguinte forma: $34 \%$ da nota referentes ao pavimento da calçada, avaliado se está em bom ou mau estado de conservação, 33 \% referentes à existência de desníveis, como por exemplo, rampas muito íngremes, e os outros $33 \%$ referentes a obstáculos existentes na calçada que possam atrapalhar a locomoção do usuário, ou seja, buracos, barreiras e outros.

\subsubsection{Gestantes e idosos}

\section{A. Número de vagas específicas no estacionamento}

A Lei Federal 10.741/2008 define que $5 \%$ das vagas regulamentadas de uso público devem ser destinadas aos idosos. $\mathrm{O}$ cálculo foi feito da mesma forma apresentada para os usuários sem bagagem.

\subsubsection{Estrangeiro}

\section{A. Sinalização}

É verificado se existe sinalização indicativa em inglês e se ela é suficiente para um motorista estrangeiro se localizar, caso esse não conheça o aeroporto, utilize um carro alugado e não fale português. Aeroportos que possuem esses requisitos são avaliados com 1 (um) e aeroportos que não possuem com 0 (zero).

\section{B. Atendimento em inglês}

Nas empresas de táxi e locadoras que atendem o aeroporto é importante que existam funcionários que falem inglês, para facilitar a comunicação com os turistas estrangeiros. Aeroportos que possuem atendimento em inglês são avaliados com 1 (um) e aeroportos que não possuem, com 0 (zero).

\subsubsection{Cadeirante}

\section{A. Meio-fio}

Para as pessoas portadoras de deficiência, a NBR 14273 ACESSIBILIDADE DA PESSOA PORTADORA DE DEFICIÊNCIA NO TRANSPORTE AÉREO COMERCIAL - define que deve existir, no mínimo, uma vaga destinada a veículos que estejam sendo utilizados para o transporte da pessoa portadora de deficiência. A análise dessa vez foi feita pelo número de vagas no meio-fio. Foi verificado o número de vagas destinadas a pessoas portadoras de deficiência que chegam de táxi ou carona.

\section{B. Distância do ponto até o terminal de passageiros}

As vagas para embarque e desembarque para cadeirantes no meio-fio que utilizam táxi ou carona até o terminal de passageiros devem estar o mais próximo possível das portas de entrada. A distância dessas vagas foi calculada da mes- ma forma que aquela apresentada para passageiros sem bagagens. Nos aeroportos em que não existem vagas específicas para portadores de deficiência utilizando táxi, essa distância não foi computada.

\section{Rebaixo nas vagas do meio-fio}

Os meio-fios dos aeroportos foram avaliados com a nota máxima 1 e a mínima 0 . Para análise desse indicador nos aeroportos, foram dadas notas para os requisitos da seguinte maneira: $50 \%$ para a existência de rebaixos nas vagas de portadores de mobilidade reduzida, $40 \%$ para as condições de conservação dos rebaixos e $10 \%$ para a existência de corrimões.

De acordo com a NBR 9050 - ACESSIBILIDADE A EDIFICAÇÕES, MOBILIÁRIO, ESPAÇOS E EQUIPAMENTOS URBANOS, a existência dos corrimões para as vagas destinadas aos portadores de mobilidade reduzida não é obrigatória, por esse motivo sua nota de avaliação é menor.

\section{Número de vagas específicas no estacionamento}

A Lei Federal 10.098/2000 define, no parágrafo único de seu sétimo artigo, que as vagas deverão ser em número equivalente a dois por cento do total, garantida, no mínimo, uma vaga. Foi adotado esse parâmetro para o cálculo de vagas de usuários com mobilidade reduzida. O cálculo foi feito da mesma forma apresentada para os usuários sem bagagem.

\section{E. Entrada e saída adaptada}

Tanto a entrada como a saída do estacionamento devem ser adaptadas de modo que os usuários com restrições de mobilidade não sejam impedidos de se movimentar devido a degraus, rampas e demais obstáculos, permitindo a livre circulação. Essas entradas e saídas devem possuir rampas ou rebaixos, fazendo a concordância de nível do estacionamento com a calçada que leva o passageiro até o terminal.

Nesse indicador foi verificado se as entradas e saídas do estacionamento são adaptadas para usuários com restrições de mobilidade. As entradas foram avaliadas, com a nota máxima sendo 1 (um) e a mínima 0 (zero). Para análise desse indicador nos aeroportos, foram dadas notas para os requisitos da seguinte maneira: $40 \%$ para a existência de rebaixos, $40 \%$ se estiver adequado à norma NBR 9050, de 2004, e 20 \% para as condições da pavimentação.

\section{F. Veículo adaptado para cadeirante}

Os veículos adaptados a cadeirantes devem possuir um bagageiro que acomode bem a cadeira de rodas e é importante que o acesso a cabine seja fácil. Existem veículos que possuem uma plataforma elevatória automática para facilitar a entrada e saída do cadeirante.

Para aluguel de carros é importante que o veículo possua câmbio automático e todos os comandos adaptados para que o usuário possa dirigir usando apenas as mãos. Os aeroportos que possuem empresas de táxis ou locadoras que possuem veículos adaptados foram pontuados com 1 (um) enquanto os que não possuem com 0 (zero).

\subsubsection{Deficiente visual}

\section{A. Linha guia ou piso tátil}

Para facilitar a identificação do percurso de pessoas com deficiência sensorial visual, o aeroporto deve possuir uma linha guia ou piso tátil. Estes podem ser elementos natu- 
rais ou edificados que possam ser utilizados como guia de balizamento para pessoas com deficiência visual que utilizem bengala de rastreamento. As faixas no piso devem possuir textura e cor diferenciadas. Os aeroportos que possuem linha guia ou piso tátil foram pontuados com 1 (um) enquanto os que não possuem com 0 (zero).

\subsection{Construção do Índice de Acessibilidade}

A estruturação do índice se deu por diferentes níveis, conforme a Figura 2. No superior, foco de atenção primária, encontra-se o tipo de usuário. No seguinte estão os modos de transporte disponíveis para acesso ao aeroporto. No inferior encontram-se os Indicadores - específicos para cada tipo de usuário e cada modo de transporte. Todos os elementos foram analisados e agregados, a fim de sintetizar as informações específicas e chegar-se a uma nota final que permita confrontar os diferentes níveis de acessibilidade dos aeroportos estudados.

\subsection{Pesos dos indicadores}

A ponderação da importância relativa de cada um dos indicadores é uma tarefa que requer atenção especial. De um modo geral depende do ponto de vista do analista e, deste modo, apresenta uma série de perspectivas diferentes, que podem até ser complementares. Uma abordagem comumente utilizada para esta distribuição de pesos é a "Método de Análise Hierárquica" (ou AHP, do original em inglês Analytic Hierarchy Process), técnica que utiliza critérios múltiplos. Esta metodologia possibilita considerar conjuntamente dimensões distintas, permitindo a realização de avaliações em bases múltiplas. Para garantir a necessária consistência ao sistema de pesos, deve-se levar em conta que o aumento de influência em alguma dimensão deve ser compensado pela diminuição na de outra(s), ou seja, a soma dos pesos atribuídos a mesma categoria deve, necessariamente, ser igual à unidade.

Além disto, para cada tipo de usuário, os indicadores possuem um peso diferente de acordo com a sua utilidade específica, refletindo no modo de transporte que ele utilizou no acesso ao aeroporto. Deste modo, existe uma segunda ponderação. Estes pesos podem ser obtidos de diversas formas (inclusive ouvindo os usuários, ou especialistas), mas aqui ela é simplesmente a divisão da unidade respectiva de cada indicador pela soma do número de indicadores relacionados ao tipo de usuário e ao modo de transporte. Isto se dá de tal modo que para um mesmo meio de transporte e mesmo tipo de usuário os pesos sejam distribuídos igualmente e a soma de todos eles seja 1.

\subsection{Normalização das notas}

Depois de definidos os pesos dos indicadores que compõem o índice, a etapa posterior consiste na definição do processo de normalização destes valores. Esse processo é importante, já que os valores encontrados caracterizam diferentes critérios, não comparáveis entre si e representados por diferentes escalas. Dessa forma, eles foram normalizados para uma mesma escala de valores, para posteriormente serem agregados através de um modelo matemático. Para a normalização das notas dos indicadores foi definido o limite mínimo e máximo de zero e um, respectivamente.

\subsection{Agregação dos indicadores}

Uma vez normalizados os indicadores para valores entre zero e um, a etapa seguinte consistiu na sua agregação de forma a chegar-se ao índice global. $\mathrm{O}$ método de agregação do Índice de Acessibilidade consistiu em uma estrutura linear ponderada, onde os critérios são combinados através de uma média ponderada, permitindo a compensação entre os mesmos. Esta combinação é feita através de quatro equações. A primeira é aplicada para se obter a nota específica para cada tipo de usuário, realizando o somatório das notas normalizadas dos indicadores multiplicadas pelos pesos referentes a eles como é mostrado na equação (2).

$$
N_{1}=\sum w_{i} \cdot n_{i}
$$

em que,

$$
\begin{aligned}
N_{1}: & \text { Nota } 1 \text { para cada tipo de usuário; } \\
w_{i}: & \text { peso do indicador } i ; \text { e } \\
n_{i}: & \text { nota (valor normalizado) obtida para o indicador } i .
\end{aligned}
$$

A segunda equação é aplicada para ponderar a participação de cada tipo de transporte na nota final. Para essa ponderação foram utilizadas as pesquisas de origem e destino (OD) realizadas pela FIPE, nas quais a participação de cada um dos modos de transporte era especificada. Assim, cada um deles recebeu o peso de acordo com o seu uso, como na equação (3). As notas obtidas acima para cada tipo de transporte são, então, somadas para chegar-se a uma nota final para cada tipo de usuário por aeroporto estudado (equação 4).

$$
N_{2}=\sum N_{1} \cdot m
$$

em que,

$$
\begin{aligned}
N_{2}: & \text { Nota } 2 \text { para cada tipo de usuário; e } \\
m: & \text { peso do modo a que pertence a Nota } 1 .
\end{aligned}
$$

$$
N F=\sum N_{2}
$$

Finalmente, para a nota final do Índice de Acessibilidade foi feito o somatório das notas encontradas para cada tipo de usuário e dividido por seis (6), número de tipos de usuários avaliados (equação 5). Os resultados com as notas finais dos aeroportos são mostrados no próximo item.

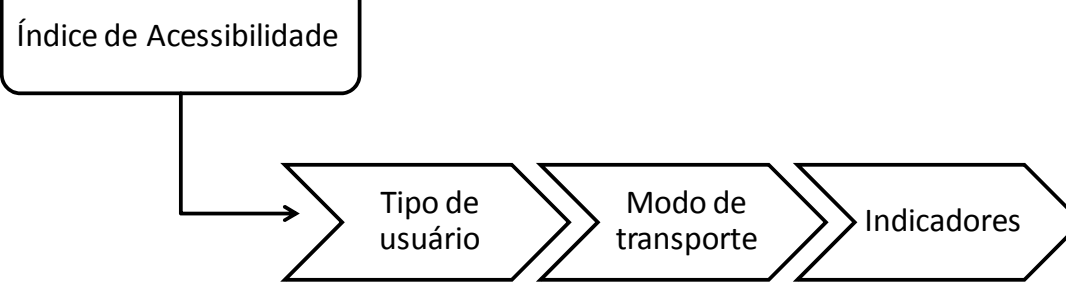

Figura 2. : Níveis de estruturação do Índice de Acessibilidade 


$$
I A=\frac{\sum N F}{6}
$$

\section{RESULTADOS}

Neste item são apresentados os resultados obtidos para o Índice de Acessibilidade dos aeroportos selecionados. Uma vez que os limites mínimo e máximo do Índice de Acessibilidade situam-se entre os valores zero $(0,00)$ e um $(1,00)$, resultados finais próximos a zero indicam desempenho ruim do aeroporto em relação à acessibilidade do usuário. Já valores próximos a um mostram que o aeroporto analisado apresenta boas condições de acessibilidade para os diversos perfis de usuários considerados. É importante observar que os resultados apresentados a seguir são reflexos da ponderação dada aos indicadores e explicada no item anterior. Se o foco da avaliação fosse outro, os indicadores poderiam ser calibrados e os resultados seriam diferentes.

O resultado global do Índice de Acessibilidade para os seis aeroportos estudados, obtido através do processo de cálculo dos indicadores, é apresentado na Figura 3. O gráfico apresentado nesta figura evidencia que os aeroportos estudados apresentaram resultados com uma amplitude de 0,15 (variação de 0,465 até 0,625 ) na escala de avaliação do índice, que tem amplitude total de 1,00. O aeroporto Santos Dumont, no Rio de Janeiro, foi o que obteve a maior pontuação, com valor 0,625 . O valor obtido não chega a ser baixo. Indica, porém, que vários aspectos ainda podem ser melhorados para se obter uma melhor pontuação e, consequentemente, melhores resultados em termos de acessibilidade do aeroporto. Os aeroportos de Guarulhos, em São Paulo, e Juscelino Kubitscheck, em Brasília, apresentaram valores muito próximos e obtiveram os piores resultados dentre os seis aeroportos analisados, 0,465 e 0,470, respectivamente.

De modo geral, nenhum dos aeroportos estudados obteve pontuações altas, mostrando que ainda há muitos aspectos que devem ser melhorados para a acessibilidade e conforto dos diversos tipos de passageiros. A comparação entre os indicadores analisados de cada aeroporto pode servir como um bom guia para a melhora das condições desses e de outros aeroportos.

\subsection{Resultados por tipos de usuários}

A Figura 4 permite visualizar os resultados obtidos para cada perfil de usuário considerado. Cada linha fechada representa um aeroporto e aqueles que possuem suas linhas mais próximas dos limites externos do gráfico são os que obtiveram melhores avaliações. É importante observar que a escala do gráfico varia, neste caso, apenas de 0,00 até 0,70 . Como a escala de pontuação tem um valor máximo possível igual a 1,00 , isto indica que nenhum aeroporto estudado obteve a pontuação máxima, para nenhum tipo de usuário. Para os passageiros com e sem bagagem, os índices encontrados para um tipo de passageiro são praticamente os mesmos encontrados para o outro. Para eles, o aeroporto de Guarulhos apresentou, em ambos os casos, os piores resultados, com valores no patamar de 0,4 . No outro extremo, os aeroportos Santos Dumont e Viracopos apresentaram os maiores valores, com resultados muito próximos.

Analisando o gráfico da Figura 4 fica também evidente como os aeroportos estudados são pouco preparados para receber passageiros com deficiência visual. $\mathrm{O}$ aeroporto que obteve a maior pontuação para esse tipo de usuário foi o de Congonhas, mas com um valor que não chega a atingir o patamar de 0,60. Os aeroportos de Brasília, Galeão e Viracopos obtiveram valores ainda menores para esses usuários, sendo Brasília o pior de todos. Esses mesmos aeroportos também não se mostraram preparados para receber passageiros idosos, diferentemente dos aeroportos de Congonhas, Santos Dumont e Viracopos, que apresentaram valores substancialmente maiores que os anteriores para esse perfil de usuário.

No conjunto dos aeroportos estudados, a curva do aeroporto de Congonhas se destaca para usuários idosos, cadeirantes e com deficiência visual. Este é o aeroporto que se mostra mais preparado para receber esses perfis de passageiros, embora ainda esteja longe do valor máximo da escala, que vai até 1,0 (um). Para estrangeiros que chegam ao país, o aeroporto Santos Dumont é o que tem as melhores condições para atendê-los e apresenta uma nota bastante superior aos demais. O aeroporto de Guarulhos é o que apresenta a menor nota, um pouco acima de 0,40 , para esse tipo de usuário. Este pode ser um problema sério para este aeroporto, já que ele é uma das principais portas de entrada para estrangeiros no país e deveria estar muito bem preparado para receber estrangeiros. Os demais aeroportos apresentam notas semelhantes, que se situam entre 0,50 e 0,60 .

Índice de Acessibilidade

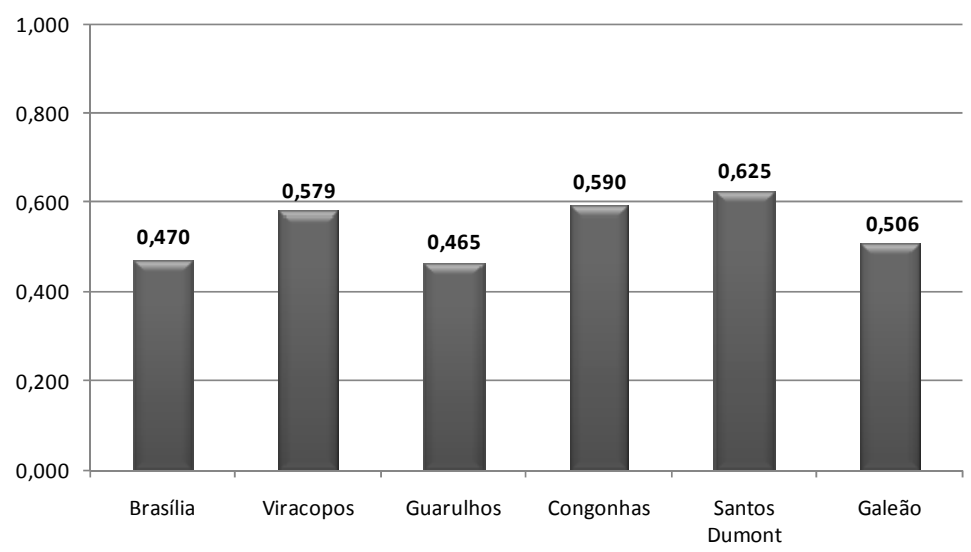

Figure 3. Valores globais do Índice de Acessibilidade para os aeroportos selecionados 


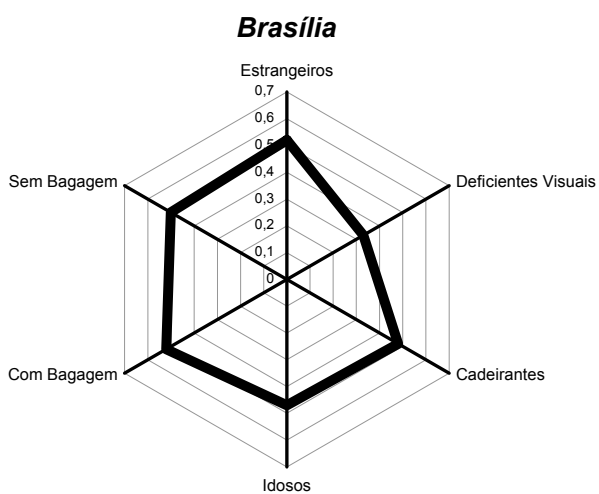

Guarulhos

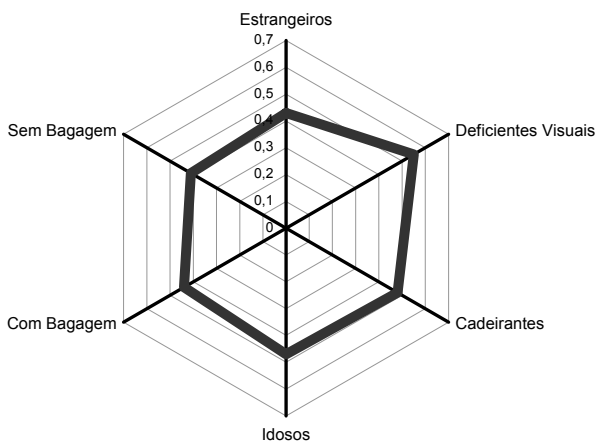

Santos Dumont

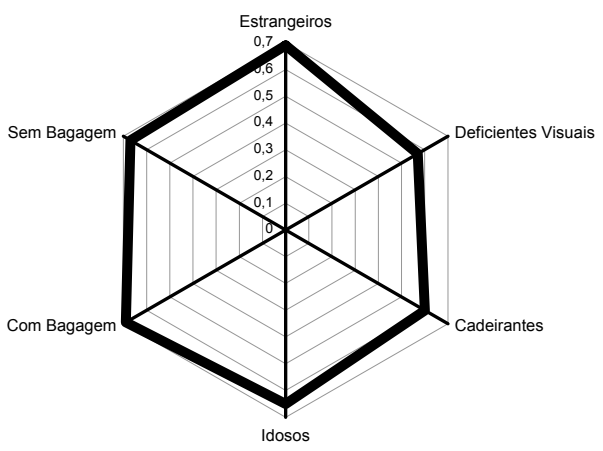

Viracopos

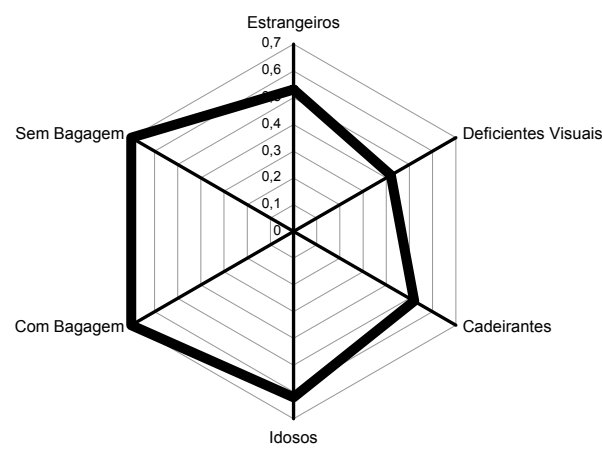

Congonhas

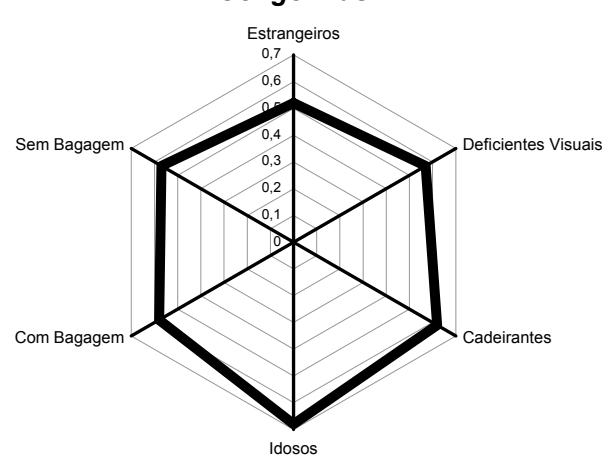

Galeão

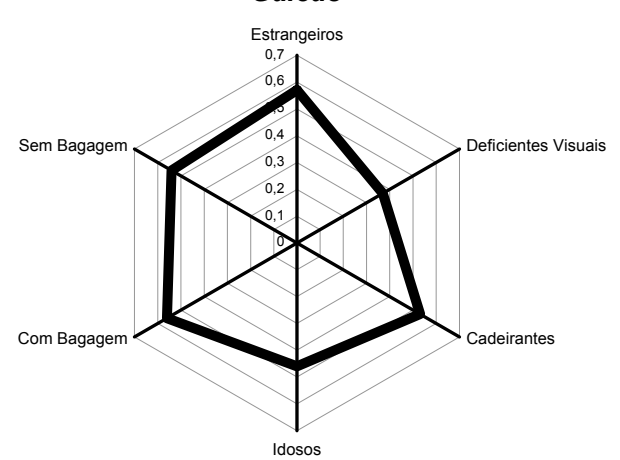

Figura 4. Valores do Índice de Acessibilidade dos diferentes perfis de usuários

\section{CONCLUSÃO}

Partindo-se de um posicionamento inicial de analisar a questão pela perspectiva do usuário, buscou-se atingir o objetivo estabelecido para o trabalho, que era a criação e aplicação de um índice de acessibilidade de aeroportos com base em aspectos objetivos, quantificáveis, que permita fazer uma comparação direta entre os terminais estudados. Em seguida, ao procurar quantificar cada um dos parâmetros de medida utilizados, buscou-se o desenvolvimento de uma metodologia que sirva como instrumento para avaliar a acessibilidade dos aeroportos e também permita o estabelecimento de planos de ação que venham, setorialmente, melhorar o seu desempenho. O desenvolvimento e aplicação do índice a seis importantes aeroportos brasileiros resultou em algumas conclusões, tanto no que diz respeito aos resultados da aplicação quanto ao método em si.

O método não busca fazer um posicionamento absoluto, mas sim uma avaliação relativa partindo do aeroporto, dos seis incluídos no processo, que melhor atenda a um determinado indicador e adotando-o como pontuação máxima neste indicador. Naturalmente, com a alteração dos aero- portos considerados, seja por inclusão seja por exclusão, as pontuações seriam alteradas. Também a outra ponderação entre os indicadores, e mesmo a sua modificação, sempre antecedida por uma calibração específica, pode ser considerada dentro da metodologia proposta sem que se perca consistência. Embora a nota final do índice seja a classificação geral em relação à acessibilidade dos aeroportos, o método também permite a avaliação setorial, de forma que possam ser avaliadas as condições de mobilidade de cada tipo de usuário ou de cada meio de transporte de acesso ao aeroporto.

A aplicação do método nos seis aeroportos estudados possibilitou verificar os níveis de serviço oferecidos aos usuários em cada um deles e identificar quais aspectos devem ser revistos e re-projetados. Os aeroportos estudados apresentaram notas gerais entre 0,465 e 0,625 , sendo o aeroporto Santos Dumont, no Rio de Janeiro, o que obteve a maior pontuação e o aeroporto de Guarulhos, em São Paulo, o que obteve o pior resultado. De modo geral constatouse que os terminais apresentam problemas para atender os requisitos mínimos de certos tipos de usuários. É o caso de passageiros com deficiência visual. $\mathrm{O}$ aeroporto que obteve a maior pontuação foi o de Congonhas, mas com uma nota 
tão baixa que não chega a atingir o patamar de 0,60 . A curva do aeroporto de Congonhas se destaca de forma positiva para usuários idosos, cadeirantes e com deficiência visual. Este é o aeroporto que se mostra mais preparado para receber esses tipos de passageiros.

A análise feita tem o objetivo de servir como um guia para que os responsáveis possam ver com clareza os aspectos a serem melhorados no acesso aos aeroportos que conduzam a um maior conforto aos usuários. É importante lembrar que essa ferramenta pode ser especialmente útil nesse momento em que três dos aeroportos estudados (Viracopos, Guarulhos e Brasília) foram concedidos pela Infraero no intuito de acelerar as obras necessárias no atendimento da demanda esperada por conta da Copa do Mundo em 2014 e dos Jogos Olímpicos em 2016. Estes são eventos de porte internacional que contam com um público específico, exigente, com enorme participação estrangeira e que estará entrando em uma região desconhecida. O Brasil terá dois anos para se preparar. Em nenhum momento pretendeu-se, no entanto, esgotar o tema, mas sim participar de um processo inicial de estudos da área que apenas começa a ficar visível nos poucos estudos relacionados ao assunto.

\section{AGRADECIMENTOS}

Os autores agradecem ao CNPq (Conselho Nacional de Desenvolvimento Científico e Tecnológico) pelo apoio.

\section{REFERÊNCIAS BIBLIOGRÁFICAS}

Alves, B. B. (2005) A importância da variabilidade do tempo de viagem no acesso terrestre a aeroportos: estudo de caso do Aeroporto Internacional André Franco Montoro. Dissertação (mestrado). EPUSP, São Paulo.

ABNT (1999) NBR 14273: Acessibilidade da pessoa portadora de deficiência no transporte aéreo comercial. Associação Brasileira de Normas Técnicas. Rio de Janeiro.

Braga, A. F. e E. P. Guedes (2008) Proposta de Cálculo da Acessibilidade em Aeroportos com o uso de Sistema de Informações Geográficas. Anais do XXII Congresso de Pesquisa e Ensino em Transportes, ANPET, Fortaleza-CE.

Caves, R. E. e G. D. Gosling (1999) Strategic Airport Planning. 1. ed. Oxford: Elsevier Science Ltd.

Clark, M. C. e W. H. K. Lam (1990) Airport ground access considerations. Airports into the 21st Century. Hong Kong Institution of Engineers, Hong Kong, 5-7, Fevereiro.

FAA - Federal Aviation Administration (1988) Planning and design guidelines for airport terminal facilities. Advisory Circular $\mathrm{N}^{\circ}$ 150/5360-13, Washington, D.C.

FIPE - Fundação Instituto de Pesquisas Econômicas (2009) Caracterização e Dimensionamento da Matriz Origem Destino do Transporte Aéreo no Brasil - 2009: Total Brasil. São Paulo. 118 p.

Gosling, G. D. (1997) Airport Ground Access and Intermodal Interface. Transportation Research Record, n. 1600, p. 10-17. DOI: 10.3141/1600-02.

Gosling, G. D. (2008) Airport Ground Access Mode Choice Models, a synthesis of airport practice. Airport Cooperative Research Program synthesis 5, Transport Research Board. Disponível em: $<$ http://onlinepubs.trb.org/onlinepubs/acrp/acrp_syn_005.pdf >. (Acesso em 10/07/2012).

Harvey, G. (1986) Study of airport access mode choice. Journal of transportation Engineering. v. 112, n. 5, p. 525-545. DOI: 10.1061/ (ASCE)0733-947X(1986)112:5(525).

IATA - International Air Transportation Association (1989) Airport Terminal Reference Manual. $7^{\text {th }}$ Edition, Montreal.

Koster, P.; E. Kroes e E. T. Verhoef (2010) Travel Time Variability and Airport Accessibility. Tinbergen Institute Discussion Paper. TI 2010-061/3.

Kouwenhoven, M. (2008) The Role of Accessibility in Passengers' Choice of Airports, OECD. Discussion Paper n. 2008-14, Paris. DOI: $\underline{10.1787 / 9789282102466-5-\mathrm{en}}$.
Kouwenhoven, M.; E. Kroes e J. Veldhuis (2008) Forecasting the impact of a ticket tax in the Netherlands. European Transport Conference, Noordwijk.

Mamede, D. A. e C. J. P. Alves (2009) Estudo sobre a acessibilidade de aeroportos no Brasil. Anais do $15^{\circ}$ Encontro de Iniciação Científica e Pós-Graduação do ITA - XV ENCITA / 2009. Instituto Tecnológico de Aeronáutica, São José dos Campos, SP, Brasil, Outubro, 19 a 22.

McKinsey \& Company (2010) Estudo do Setor de Transporte Aéreo do Brasil: Relatório Consolidado. Rio de Janeiro.

Monteiro, A. B. e M. Hansen (1996) Improvements to Airport Ground Access and Behavior of Multiple Airport System: BART Extension to San Francisco International Airport. Transportation Research Record, n. 1562, p. 38-47. DOI: 10.3141/1562-05.

Skinner, R. E. (1976) Airport choice: an empirical study. Transportation Engineering Journal, v. 102, v. 4, p. 871-882. 